been on a diet, $82.1 \%$ eat vegetables less than once a day. Of those who smoke, $62.5 \%$ eat fruit less than once a day. All the women who have a body mass index $>25$ eat vegetables less than once a day and $45.5 \%$ do not have breakfast. Among those who do not have breakfast, $83.8 \%$ eat vegetables less than once a day. The group of women from North Africa was too small for statistical analysis.

In conclusion, the dietary habits of these underprivileged women are characterized by disturbances of their dietary rhythms and dietary balance. Bad eating habits are more common among those who smoke, have already followed a diet or are overweight.

Effects of nutrition education on magnesium intake during pregnancy. JM Lecerf ${ }^{1}, \mathrm{C}$ Pavy ${ }^{2}$, M Delcroix ${ }^{2}$ ( ${ }^{1}$ Institut Pasteur de Lille, service de nutrition, 1, rue du Professeur-Calmette, 59019 Lille cedex; 2 Hôpital Saint-Philibert, 59160 Lomme, France)

Magnesium deficiency during pregnancy can be induced by an inadequate intake and can have some maternal or fetal outcomes. It was decided to assess the magnesium intake of pregnant women and to increase this amount through nutrition education.

A group of 30 randomly selected pregnant French women in their second trimester of pregnancy were given a dietary inquiry on a week-day and on a week-end-day (24 $h$ recall) and a food frequency questionnaire about magnesium-rich foods, in order to evaluate their initial magnesium intakes. Advice and information were given simultaneously about magnesium in the diet. Three weeks later a new dietary intake questionnaire was given on $2 \mathrm{~d}$. A third and second food frequency record were given 3 weeks later at 6 weeks.
The mean magnesium intake was initially (Q1) $280 \pm 93 \mathrm{mg} / \mathrm{d}$ (recommended dietary allowances $480 \mathrm{mg} / \mathrm{d}$ ). Three weeks after having received the nutritional advice the mean magnesium intake (Q2) was $325 \pm$ $88 \mathrm{mg} / \mathrm{d}$ and at 6 weeks (Q3) it was $317 \pm$ $102 \mathrm{mg} / \mathrm{d}$. The difference was significant between Q1 and Q2 $(p<0.01)$ and Q1 and Q3 $(p<0.05)$ but there was no difference between Q2 and Q3. Simultaneously the caloric intake did not increase significantly, the carbohydrate intake increased and the lipid intake decreased. At 3 weeks, 21 women increased their magnesium consumption and 20 at 6 weeks. Among those who increased their magnesium consumption, the mean intake increased from $256 \pm 79 \mathrm{mg} / \mathrm{d}$ to $340 \pm 92 \mathrm{mg} / \mathrm{d}$ and then to $361 \pm 105 \mathrm{mg} / \mathrm{d}$. The food frequency record for the whole group of women revealed that 21 increased their intake of cooked vegetables, 15 raw vegetables and 19 bread. A greater number ate legumes, wholemeal bread, and fewer boiled foods.

In conclusion, the magnesium intake of the investigated pregnant women was insufficient and increased significantly after they received a nutrition education. It remained, however, lower than recommended dietary allowances.

\section{DIET AND DIGESTION}

Characterization of dietary fat emulsification in the digestive tract of healthy human subjects. $M$ Armand ${ }^{1}, P$ Borel ${ }^{1}, C$ Dubois ${ }^{1}, \mathrm{M}$ Senft ${ }^{1}$, J Peyrot ${ }^{2}$, J Salducci ${ }^{2}$, H Lafont ${ }^{1}$, D Lairon ${ }^{1}$ ( 1 Unité 130-INSERM, 18, avenue Mozart, 13009 Marseille; ${ }^{2} \mathrm{CHU}$ Nord, service d'hépato-gastro-entérologie, 13015 Marseille, France)

Fat emulsification plays a key role in dietary fat digestion by creating a lipid-water interface and thus allowing an interaction between soluble lipases and insoluble fat. 